\title{
Form-finding to fabrication of super-thin anisotropic gridshell
}

\author{
Roberto Naboni \\ ACTLAB - ABC Department, Politecnico di Milano, \\ Milan, Italy \\ roberto.naboni@polimi.it
}

\begin{abstract}
The paper discusses the process of computational design, analysis and fabrication for a lightweight super-thin gridshell structure. Digital form-finding based on Particle Spring Systems is used to define a compression-based shape, which is discretized through a parametric process into box-shaped components with embedded assembly and structural logics. Strategies to maximize the behaviour of anisotropic construction boards, double curved forms and assembly precision are described. Results are thoroughly documented to highlight the potential of the approach to rapidly build temporary gridshell structures.
\end{abstract}

Keywords: Digital Form-Finding, Particle-Spring System, Gridshell structures, Digital Fabrication, Catenary

\section{Introduction}

\section{Potential of lightweight and ultra-lightweight structures}

Lightweight structures were introduced as a field of study at the beginning of 19th century when the influence of construction engineering in architecture became more apparent. This architectural vision occurred in parallel with new understandings and innovations in many fields of science, the development of new materials as well as a novel thought process stimulated by the industrial age (Sobek, 2012). Nowadays, the accelerated development of computersupported research and analysis provide architects with a wide range of opportunities to design and study such structures. Historically, some of the pioneering masters of architecture have explored lightweight architecture. Among them, Frei Otto and Heinz Isler worked on exploiting the use of physical models to compute efficient and elegant forms, while providing exciting spaces to envelope closed or open areas (Otto, 1974; Isler, 1961). Historically, some of the pioneering masters of architecture have explored lightweight architecture. Among them, Frei Otto's and Heinz Isler's worked on exploiting the use of physical models to compute efficient and elegant forms, while providing exciting spaces to envelope closed or open areas (Otto, 1974; Isler, 1961). In recent years, lightweight structures and shelters have gained unprecedented popularity due to the opportunity of minimizing material, as well as the possibilities they open for off-site fabrication, modular assembly and a simple dismantling process. They provide efficient and sustainable spatial solutions, adaptable forms and a high level of optimization in material use and behaviour.

\section{Thin-plate gridshell structures}

Within the more general context of lightweight structures, this paper focuses on a design and construction case study for a specific typology: the thin-plate-based gridshell. This is a structural system characterized by a three-dimensional curved surface with a relatively thin section, constructed through the assembly of discrete elements (Adriaenssens et al 2014). This is a form-passive structural typology, i.e. is not actively changing its shape under varying loading conditions. It resists mainly through membrane stresses, transferring external loads to its supports predominantly with forces acting in the plane surface, with stresses of compression, or a combination of compression and tension. A good recommendation for thin shells is to be sufficiently 'thick' to carry these compressive stresses without buckling. The work described herein is focused on the development of temporary full-scale prototype developed through the study of a base component unit, that is analysed in its performance, behaviour and structural efficiency, with the perspective of being assembled within a larger structure of similar components. Pioneering work in this field has been carried out by Marc Fornes who produced several projects where hyper-thin self-supported structures are computationally designed and fabricated maximizing double curvatures of the structure and taking advantage of its structural capacities (Fornes, 2016).

\section{Background on Form-finding methods}

The design of complex shell structures necessitates a formfinding process, intended as a "forward process in which parameters are explicitly controlled in order to find an 'optimal' geometry of a structure which is in static equilibrium with a design loading" (Adriaenssens et al 2014). The common approach in the work of the above-mentioned masters of Frei Otto and Heinz Isler lies in the use of physical models to exploit the self-organization of material under forces, towards achieving stable forms (Kudless, 2011). In this way, form does not originate from an abstract concept but from the inherent capacity of a material to compute efficient forms as a result of the negotiation between external forces and the inherent properties of the material.

Generally speaking, funicular geometries offer great opportunities for the design of shells. Their name derives 


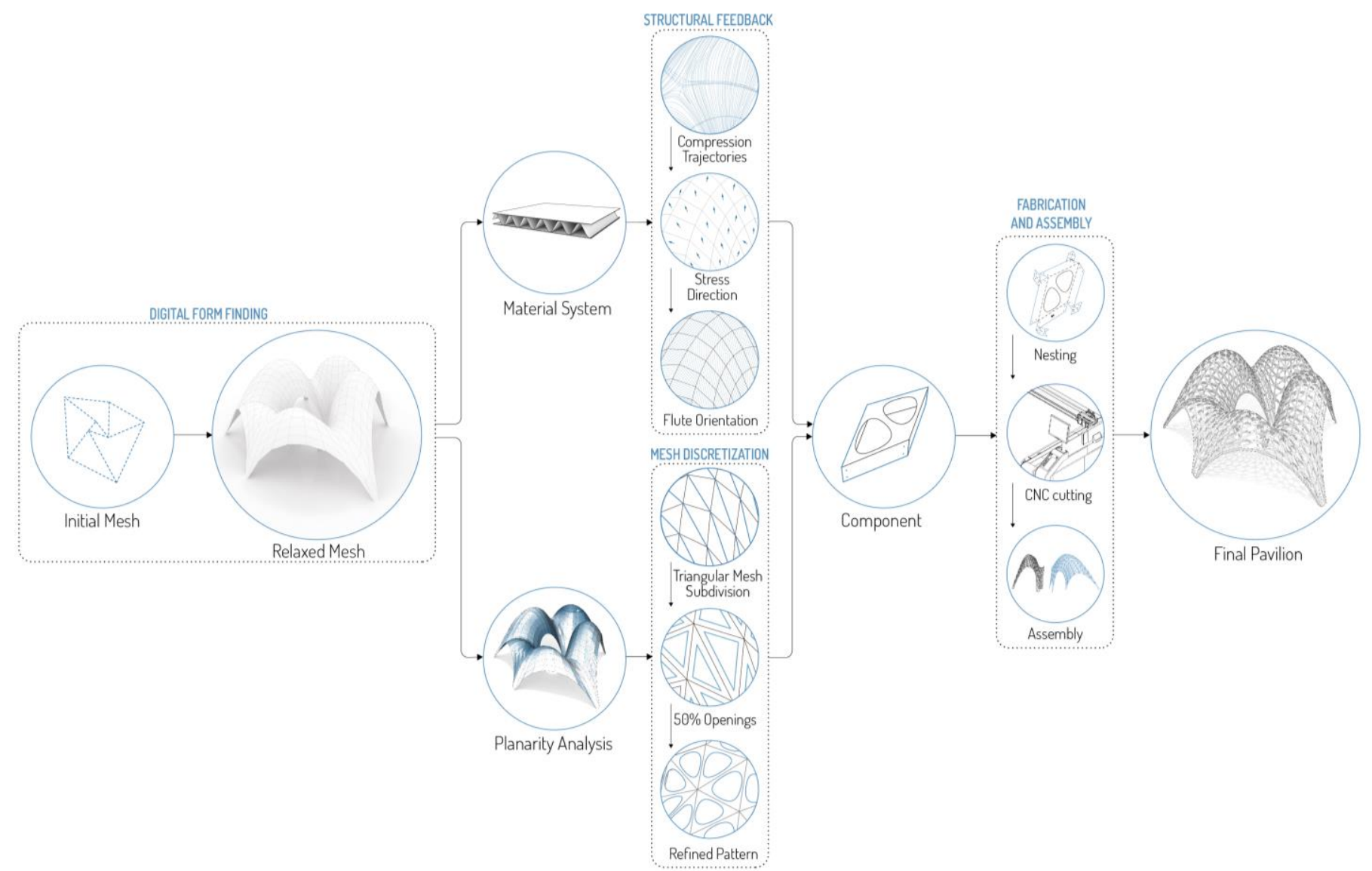

Figure 1: Diagram of the overall computational design workflow

from the Latin word funiculus, a diminutive form of funis, meaning a rope. This refers to the shape taken by a hanging chain for a given set of loads. With no flexural stiffness, the shape a cable assumes under applied loads determines 'tension-only' structures. Once an optimal shape is found, it is reversed upside down to create compression-only shells which are form-passive and are not capable of varying form according to changing loading conditions. The earliest example of funicular form finding of an arch was published by English engineer and scientist Robert Hooke (1676). 'Hooke's law of inversion' can be extended beyond a single arch and applied to the generation of shell structures with variable geometry.

At the beginning of 20th century, Antoní Gaudi employed hanging models in the form-finding processes for the chapel of the Colonia Guell and the arches of the Casa Mila (Huerta, 2006). During the 1950s catenary forms were further developed into entire shell structures. Heinz Isler employed scaled models to test thin concrete shells and to map thicknesses and locations for reinforcement, avoiding the use of sophisticated mathematical analysis. In contrast with the case of a hanging cable from two supports, hanging membranes might result in several possible forms as there are multiple possible configurations of networks for intersecting elements. Three-dimensional funicular systems are considerably more complex because of the multiple load paths that are possible (Ochsendorf \& Block, 2014). Hanging models could be used to define continuous surfaces or, as in the case of the research presented in this paper, to design discrete shells with modular elements connected at nodes.

\section{Methodological Procedures}

\section{Overview on the design process and computational workflow}

This research project implements a methodology for digital form-finding based on Particle Spring System (PSS). A selfsupported thin-plate gridshell structure has been developed through a multi step computational workflow and subsequently digitally fabricated (Figure 1). Computational and empirical studies have been conducted, with a particular focus on the structural analysis and processing of the material system, corrugated cardboard. In doing so, the following design phases have emerged:

- Digital Form Finding - involving the generation of funicular based structural morphologies, through the use of PSS. Within this phase, multiple mesh topology descriptions are tested to converge to a final description;

- $\quad$ Structural Feedback - performed through the use of FEM analysis of the self-supporting structure, where 
crucial understanding of the materials mechanical performance occurred. Principal stress trajectories and other analysis are generated;

- Component Design - the base gridshell component is tailored from a generic mesh description to a specific geometry which enhances the description of a continuous curvature, overall compressive resistance and precise assembly procedures;

- Digital Fabrication and Rapid Assembly - 528 components are produced within a considerably short time frame. The fabrication process involved the use of a large size Digital Cutter.

\section{Material system - Corrugated Cardboard}

Examples of thin plate gridshells are built with carbon fiber composites, steel and aluminium stripes or plastic plates. The material employed in this temporary structure is a corrugated cardboard KbSK342B. This unique option is convenient for testing a design methodology, as it allows easy material processing. Technically, the corrugated cardboard is a sandwich panel composed of two external face sheets, called liners, bonded to an internal wave-shaped web called fluting. The composition of the individual layers determines the overall mechanical properties. Given the singular direction flute orientation, boards have an anisotropic behaviour, extremely stiff in bending and stable against buckling in the main axis, in relation to its weight. The specific boards used in this project have an overall thickness of $3 \mathrm{~mm}$, basic weight of $402 \mathrm{~g} / \mathrm{m}^{2}$ and apparent density of $134 \mathrm{~kg} / \mathrm{m}^{3}$ as a result of a specific composition (Figure 2).

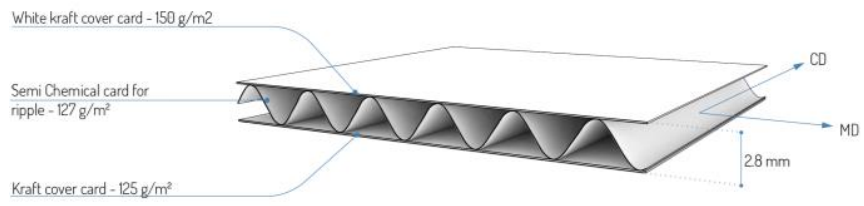

Figure 2: Section view of the material type used for the case study: $\mathrm{KbSK} 342 \mathrm{~B}, 2.8 \mathrm{~mm}$ thick corrugated cardboard with single fluting

\section{Digital form-finding}

Studies on material self-organization have vastly advanced in the last decade with the diffusion of various computational tools for digital form-finding, capable of substituting the role of physical hanging-models. Currently, the use of Particle Spring System simulations has gained popularity, following the work of early architectural exponents, Kilian and Ochsendorf (2005) which developed CADenary, a custom tool programmed in $\mathrm{C}_{++}$for virtual form-finding. The work of this paper exploits the capacity to simulate hanging chains and other force-determined shapes.

Technically, PSS involves the use of particles and springs. Particles are dimensionless points in space where all mass is concentrated. Springs connect particles to one another with straight linear elastic bars, and each spring element is assigned a constant axial stiffness, initial length and damping factor (Kilian \& Ochsendorf, 2005). The mass of particles describe the self-weight of the structural form. Once the formfinding simulation is started, particles are subjected to external forces varying their position in order to reach a shape in equilibrium. These forces are generated by the displacements of the springs from their rest length. The system oscillates, extending beyond the equilibrium shape and rebounding until a rest position is achieved, that represents an approximate equilibrium position. It is interesting to observe how this physically accurate process presents graphical analogies with the historical work of Pierre Varignon on the funicular polygon (1725) (Figure 3).
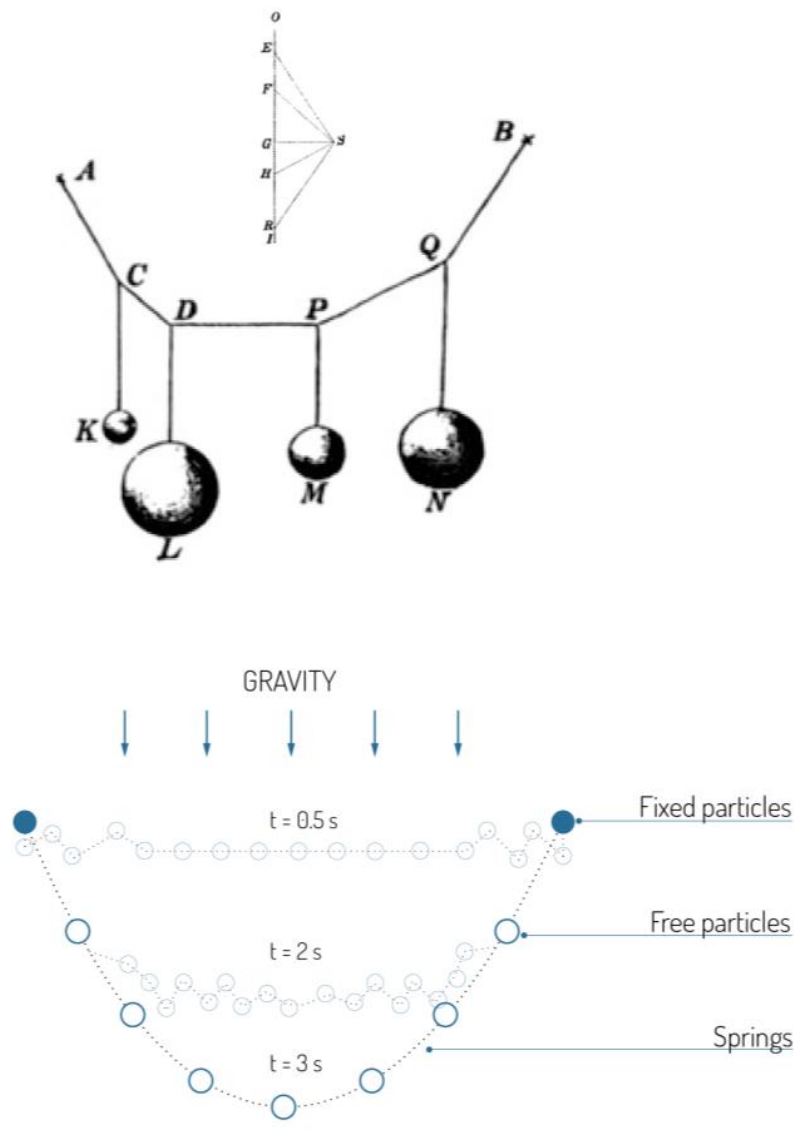

Figure 3: Comparison between the study of funicular polygons of Pierre Varignon (1725) in Nouvelle mécanique and the description of a Particle Spring System (2005).

Within this case study, the digital form-finding of the gridshell structure makes use of the widespread Kangaroo Physics for Grasshopper, a Particle Spring System created by Daniel Piker (Piker, 2010). The main advantage of this Live Physics tool is the ease of interaction between the designer and the simulation, allowing for the manipulation of points and forms, while running a physically accurate simulation. The model reacts instantaneously, allowing many design iterations in the early project stages, essentially improving the explorative potential of the form-finding process in comparison with the use of physical models.

In this form-finding process the geometry is unknown but it is generated through forces applied to an initial "matter", typically described as a mesh, which requires the definition of some arbitrary parameters such as supports, topology, and 
internal and external forces (loads). It is usually necessary to iterate several simulations in order to acquire an anticipatory intuition on the final configuration of a geometry. This process turns into an interesting negotiation between top-down designer's decision and bottom-up material self-organization. In the specific context of a design-to-fabrication workshop, this tool allowed for the exploration of many different solutions.

\section{Topology variation}

Gridshell design through form-finding is not a purely deterministic operation. Once the definition of the boundary conditions for the form-finding is established, further explorations are made in regard to the topology description of the underlying mesh. This is not to be considered as a homogeneous surface, but rather as a gridshell where the edges can converge or deviate loads, and offer higher or lower resistance to the applied loads and gravity. Given a common set of anchor points, different structural networks of interdependent catenary arches have been studied (Figure 4). In general, it has been observed that a more direct edge connectivity between anchor points generates more segmented vaulted spaces, while indirect edge connections offer more resistance to gravity and the consequential description of less variable heights. An investigation of the mesh topology is thus fundamental in characterizing the spatial configuration of a gridshell, as well as influencing the size and number of mesh faces. Therefore, fabrication constraints have to be carefully analyzed in advanced in order to optimize the final nesting efficiency. In the specific case of this research project, the criteria influencing the choice of the final topology were related to the structural and fabrication efficiency, together with a spatial analysis of the prototype in congruence with the surrounding context.

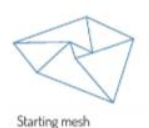

Starting mesh

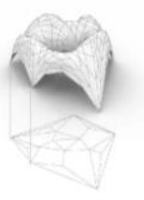

104

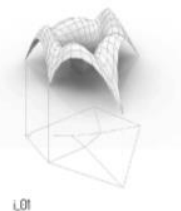

ion

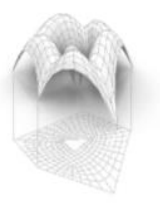

ios

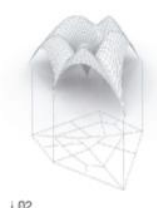

102

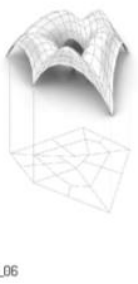

103
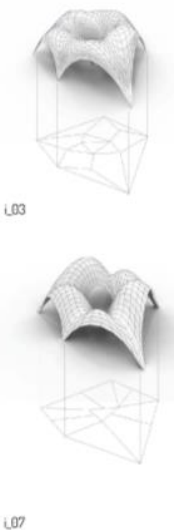

Figure 4: Iterations in the study of different mesh topologies from a common set of anchor points

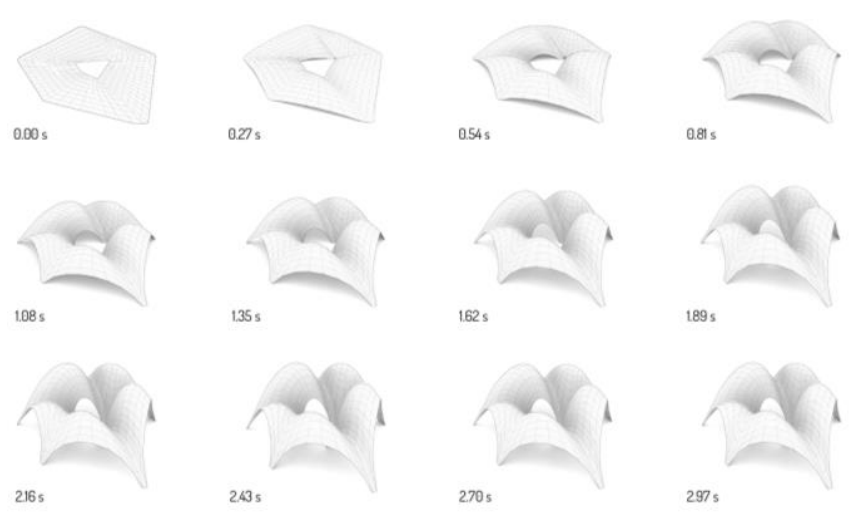

Figure 5: Simulation of the mesh relaxation process through Particle Spring System engine of Kangaroo Physics for Grasshopper

\section{Structural feedback}

The final form is analyzed through Finite Element Analysis in order to evaluate the actual mechanical behaviour, and inform the component design accordingly. This process is performed using Millipede, a specific plug-in of Grasshopper for Rhinoceros which allows quick linear elastic analysis of frame and shell elements, within the design environment. Boundary conditions, such as constraints, load, mesh thickness and material characteristics are input. Once the simulation is run, a mapped representation of Bending Moment, Deflection and Compressive Stress Lines are extracted and utilized as crucial parameters for the component development. These analyses highlight the substantial effectiveness of the form-finding process in generating a compressed structure, which present bending in correspondence with the ground constraints (Figure 6).

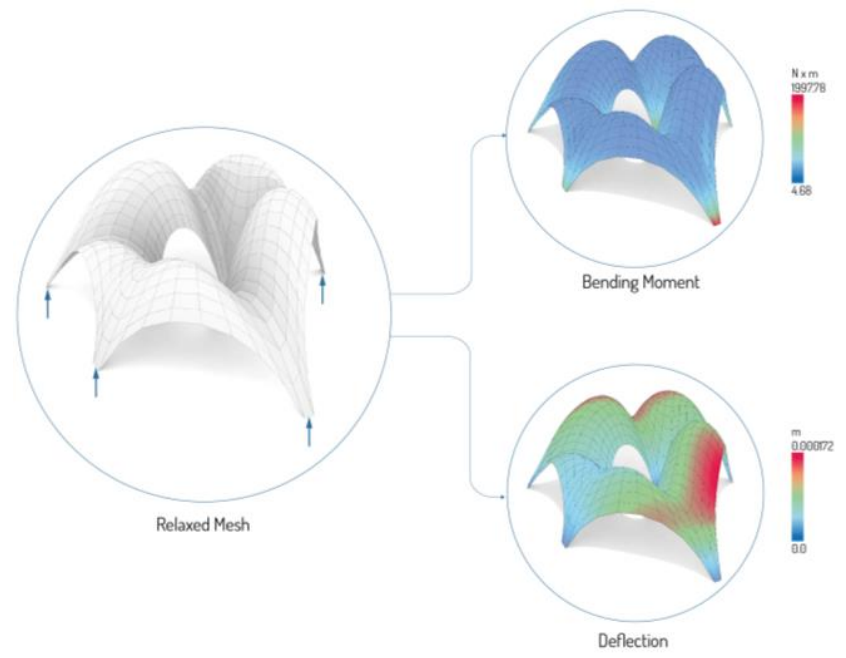

Figure 6: Finite Element Analysis of the gridshell 

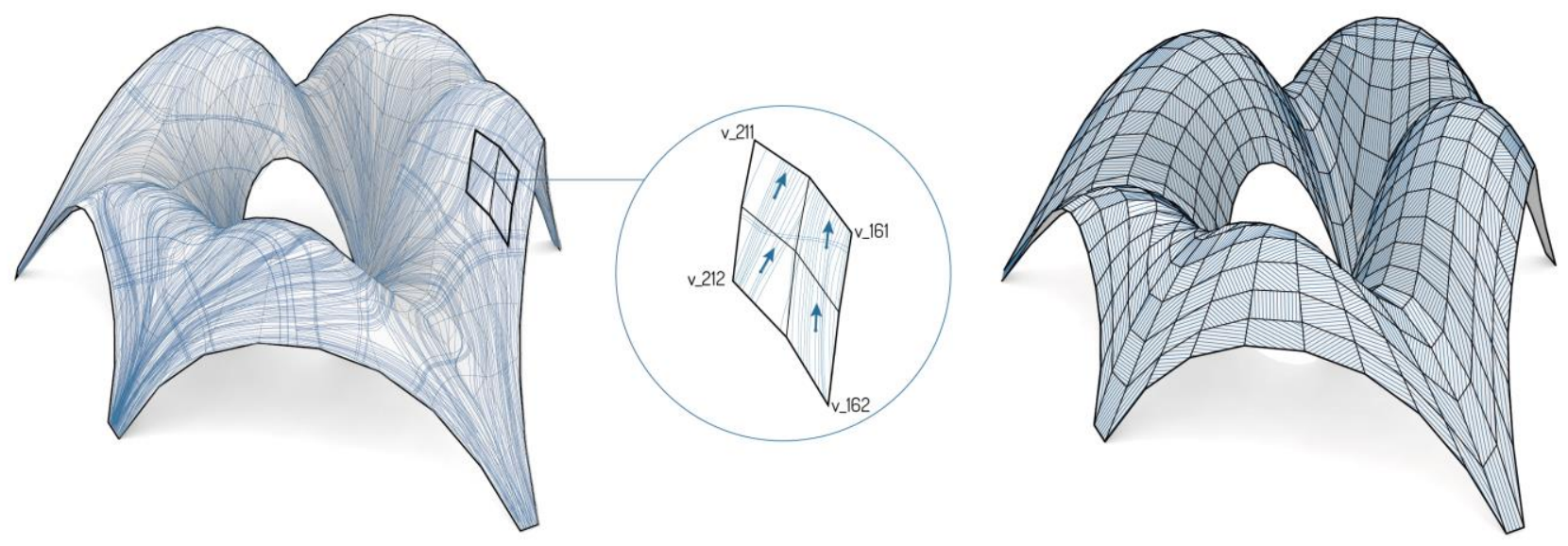

Figure 7: Scheme which shows the main compression trajectories obtained from FEM analysis, which are further used to define average vectors to inform the main fluting orientation in each gridshell component

\section{Component design}

The output of the structural analysis is used to inform the design of the base gridshell component in various ways. Firstly, the deflection analysis allows for the understanding of how the highest deflection values are found in the proximity of the midpoint of external arches. In accordance with this analysis, a reinforcement made of ribs is created in each individual component. This operation essentially transforms simple mesh quads into boxed elements that are used to interconnect the components, and locally determine the overall system precision. Secondly, the principle compression trajectories are extracted from the FEM analysis. Given that the cardboard in question is highly anisotropic, these are used to give indications on the ideal flute configuration. An average orientation vector of the compressive lines is found for each individual component. Flute direction will be placed parallel to its vector in the final nesting process.

In parallel with the structural assessment, a planarity analysis is performed on each component. Measured values proved to be limited, and the fine discretization of the shell helped to reduce curvature values without compromising the smoothness of the shell profile. A perforation pattern has been applied, allowing the components' top face to bend slightly in order to match the overall curvature. This operation not only improves the visual aesthetic of the gridshell shape, but also acts as a substantial structural operation. The accuracy of the double curved surface allows the gridshell to work with pure membrane stress.
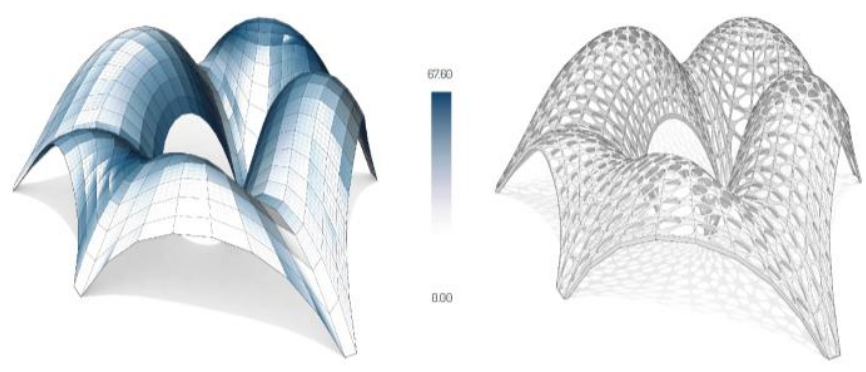

Figure 8: The representation of curvature analysis and resulting perforation in the final design to allow a correct curvature description

\section{Fabrication process and assembly}

The control over the fabrication process is of great importance for this project typology, as direct information transfer along the digital chain is needed. Fundamental material information about the component orientation is embedded as a design parameter, and the design then is prepared for fabrication, providing organized files to be machined. Each single three-dimensional component of the structure was positioned and unrolled by a specifically developed algorithm able to preserve the needed mechanic connections among the different pieces (Figure 9). Firstly aligning the main face of each component on a planar surface, and then using three dimensional rotations in order to align the construction rib-faces and joint-faces. This algorithm dramatically speeds up the operations toward the production of the pavilion, and avoids the inevitable mistakes that modelling would imply. All of the 528 gridshell components were identified by a progressive tag number which indicated the order in which the construction elements were organized.

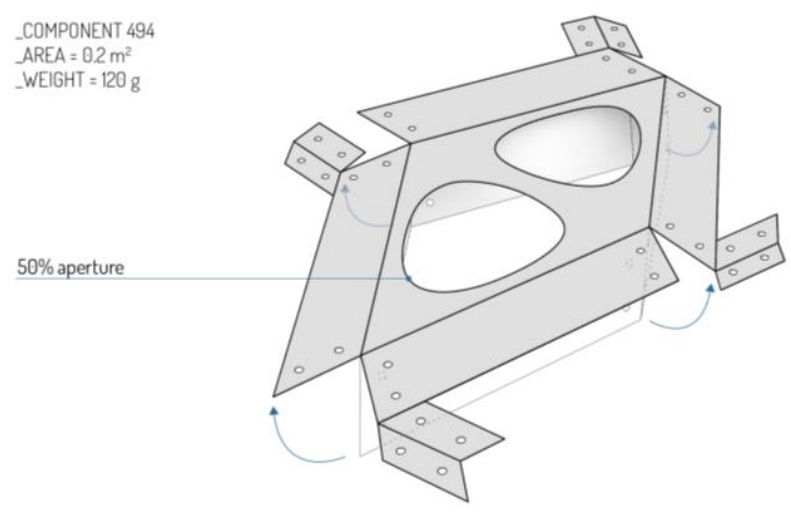

Figure 9: Schematic representation of the unrolling process performed on each component by a custom algorithm 
The three dimensional components are then transformed into 2D vectorial information which provides the digital cutting machine with geometries divided into different layers based on a full cut, half-cut, tagging and creasing. These layers were read by the CAM software which attributes different tools, heights and speed. The 528 components are then organized onto cardboard sheets with dimensions of 2400 by $1700 \mathrm{~mm}$ with the flutes oriented parallel to the main stress direction. A "performative nesting" was applied, and the components had been organized within sheets following the main criteria of flute orientation (Figure 10). The large CNC area allowed a significant minimization in material waste with an average efficiency of $85 \%$. The cardboard sheets were processed with an average speed of 50 components per hour, allowing the production of 528 components in about ten hours. The cut components were easily transported on site given their reduced weight of $38 \mathrm{~kg}$ and their extreme packing efficiency (Figure 11).

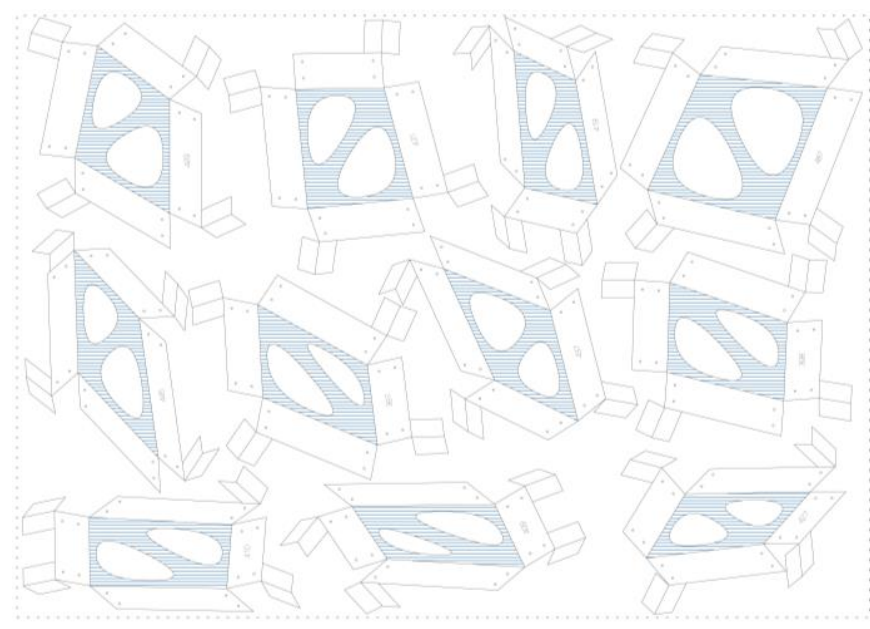

Figure 10: Performative nesting scheme showing different components' orientations according to previously defined average vector of compressive lines

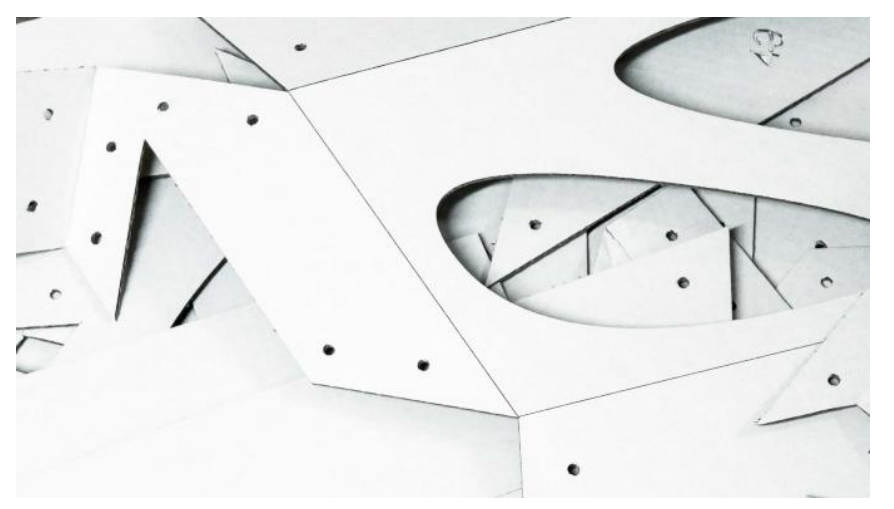

Figure 11: Gridshell components are tagged and cut with a Digital Cutting Plotter

Components were folded on site and easily fastened to acquire a stable position as boxed volumes. This process could take place without any additional measurement and without highly specialized operators since each component has joints which constrain the folding procedure into a precise position. The single components are then fastened among each other using zip-ties, composing single vaults, which were later connected together to form a self-standing structure.

\section{Results}

The paper tackles the design development of a temporary thin-plate gridshell structure. This work was the result of an interdisciplinary computational process involving different phases of design, analysis and digital fabrication. FEM analysis was implemented to determine the optimal orientation for the use of anisotropic corrugated panels with a thickness of $2.8 \mathrm{~mm}$. A set of 528 unrolled and cut components were assembled on site in three days and exhibited in the main hall of a building of the Faculty of Architecture at the Politecnico di Milano University as a temporary pavilion. The completed structure has a height of 3.5 meters and covers an area of $40 \mathrm{~m}^{2}$, allowing the free circulation of people while contributing to a new spatial configuration (Figure 12). The prototype represents a proofof-concept for thin plate based gridshells where curvature is not compromised with planarization. A combination of tessellation studies and perforation patterns permitted the description of a double-curved shell from a planar material, while preserving its mechanical features.

\section{Conclusions}

The paper provides a comprehensive computational methodology suitable for temporary shelter applications. The outlined process offers a degree of flexibility for an efficient design which can integrate various material parameters in a semi-automatic way, and consequently improve fabrication and assembly phases. Such a lightweight, super-thin gridshell structure is easily and rapidly assembled and disassembled and could be possibly integrated in any type of context, as a temporary or permanent structure. This research project suggests an alternative method of computing complex shapes by negotiating between digital and material strategies. The anisotropic nature of the material is used to provide higher structural efficiency. At the same time, gridshell components are strategically perforated to allow the material to approximate curvature values, rather than over imposing a geometrical discretization. In this sense the natural computational capacity of the material is instrumentalized to provide a smooth design. The implemented method could be used for a range of materials to fulfil various applications of shell-like shelters while only changing and adapting a few design parameters.

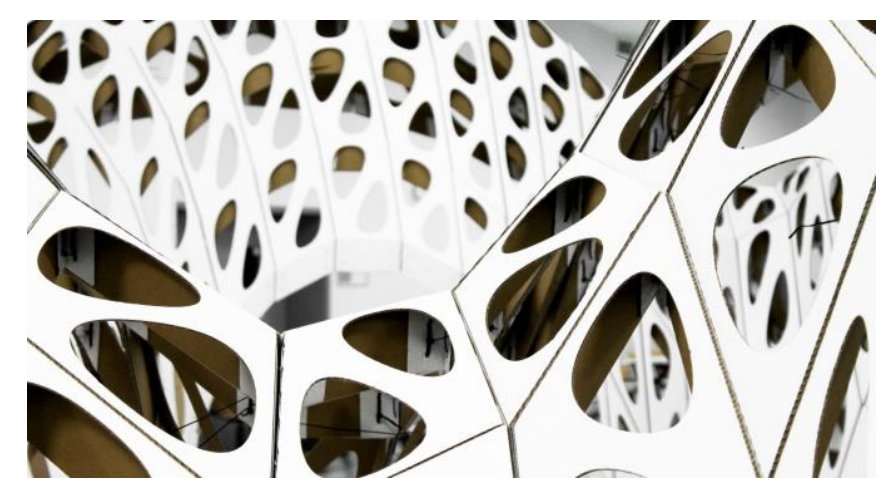




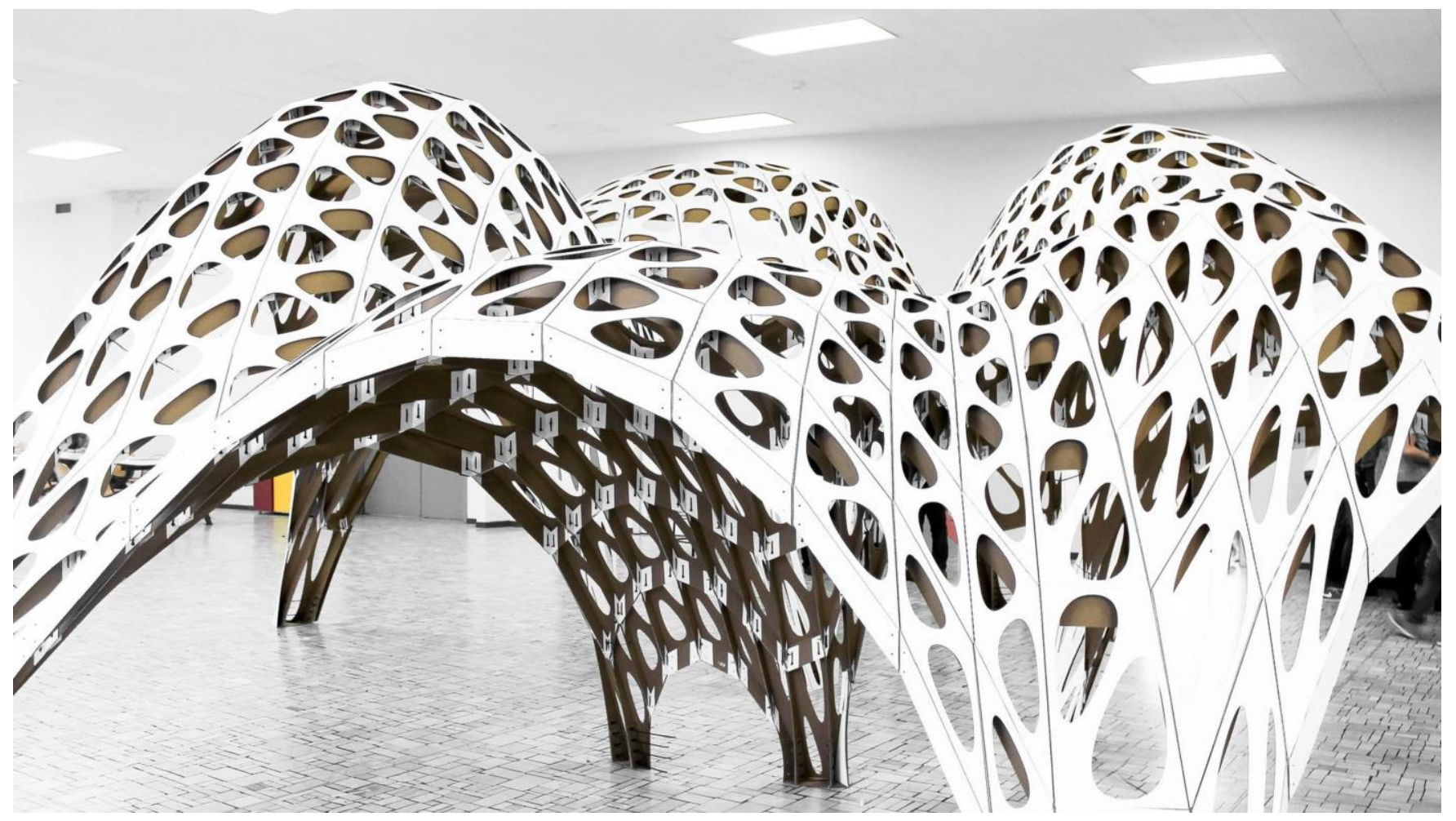

Figure 13: The final pavilion was assembled on-site in four days as a final prototype showing the utilization of digital design and fabrication methods in contemporary design

\section{Acknowledgements}

The work described in this paper is the result of different design experiments. The author would like to thank Carlo Alberto Gasparini, Paolo Alborghetti and the students participating in the UnExpected MateReality Workshop where the design and construction of the pavilion were developed: Aigerim Syzdykova, Ana Goidea, Andrea Guzzetti, Antonio Tanzini, Aziza Rustamova, Bernardo De Cossio, Cristina Manzoni, David Martinez, Francesco Roesler, Ghadeer Attallah, Giulia Maretti, Giuseppe Carbè, Julián Granados, Kalina Andreeva, Maria Vera van Embden, Marina Dimitrova, Martin Huba, Maia Zheliazkova, Michael Iannaco, Monica Cerfeda, Paolo Colombo, Paolo Franco, Sam Kakhipoor, Siamak Bazzaz, Silvia Bertolotti. Helping with construction: Lidia Korotaeva, Slavyana Popcheva. Graphic schemes included in this paper have been prepared with the help of Anja Kunic. We gratefully acknowledge Prof. Barbara Del Curto and Matteo Redaelli and Logic Srl for the use of the digital fabrication equipment.

\section{References}

Adriaenssens, S., Block, P., Veenendaal, D. \& Williams, C. (2014) Introduction, Shell Structures for Architecture: Form Finding and Optimization, pp.1-5, Taylor \& Francis Ltd.

Fornes M. (2016) The Art of the Prototypical, AD, Architectural Design, vol. 86, Issue 2, pp. 60-67.
Hooke, R. (1676) The True Theory of Elasticity or Springiness.

Kilian, A. \& Ochsendorf, J. (2005) Particle Spring Systems for Structural Form Finding, Journal of the International Association for Shell and Spatial Structures, vol. 46, No. 2, pp. 77-84.

Kudless, A. (2011) Bodies in Formation: The Material Formation of Flexible Formworks, WI: The printing house, Stoughton.

Ochsendorf, J. \& Block, P. (2014) Exploring shell forms, Shell Structures for Architecture: Form Finding and Optimization, pp. 7-12, Taylor \& Francis Ltd.

Sobek, W., Neuhäuser, S., Witte, C. \& Dr. Haase, W. (2012). Ultra lightweight construction is based on hydraulics, Institute for System Dynamics (ISYS), University of Stuttgart, Press Release, 16 April 2012.

Varignon, P. (1725) Nouvelle mécanique ou Statique, Tome 1, Chez Claude Jombert, Paris. 
SIGraDi 2016, XX Congress of the Iberoamerican Society of Digital Graphics 9-11, November, 2016 - Buenos Aires, Argentina 